\title{
Pedicled rhinotomy for clival chordomas invaginating the brainstem
}

\author{
Griff Harsh, M.D., Robert OJemann, M.D., Mark Varvares, M.D., \\ Brooke Swearingen, M.D., Mack Cheney, M.D., and Michael Joseph, M.D. \\ Stanford Brain Tumor Center and Department of Neurosurgery, Stanford Medical School, Stanford, \\ California; and Neurosurgical Service, Massachusetts General Hospital, Massachusetts Eye and Ear \\ Infirmary, and Harvard Medical School, Boston, Massachusetts
}

\begin{abstract}
Object. Clival chordomas are frequently midline lesions whose posterior growth may breach the dura and invaginate the brainstem. This precludes safe delivery of potentially curative high-dose fractionated proton radiotherapy. To avoid this problem, the authors performed pedicled rhinotomy to resect chordomas in 10 patients.

Methods. Pedicled rhinotomy is a midface transnasal route to the intercarotid sella and clivus from the tuberculum sellae to the mid-C-2 level. It involves a lateral rhinotomy incision, osteotomies of nasal bones and cartilage, lateral rotation of the nose, removal of the nasal septum and medial maxillary walls, opening of ethmoid and sphenoid sinuses, and dissection of nasopharynx and oropharynx to expose the clivus and craniovertebral junction. Tumors involving the sella, medial cavernous sinuses, middle and lower clivus, and C-1 arch and dens can be removed even if they traverse the dura. Closure involves dural repair, grafting of fat and split-thickness skin, rotation of a vascularized mucosal pedicle, and reattachment of nasal cartilage.

Ten clival chordomas in adult patients were surgically removed via a pedicled rhinotomy approach. Seven patients had previously undergone a total of nine skull base procedures. In eight of the 10 patients, tumors compressing the brainstem were completely removed using this technique. One patient required an additional subtemporal resection of a suprasellar tumor before definitive radiotherapy could be undertaken. No patient sustained any new neurological deficit; in eight patients headache, diplopia, or hemiparesis improved. One patient developed postoperative cerebrospinal fluid leakage and meningitis that were successfully treated with antibiotic agents and shunt placement.

Conclusions. Pedicled rhinotomy provides excellent shallow-field exposure of midline clival chordomas and permits relief of brainstem compression and the postoperative administration of potentially curative proton beam irradiation.
\end{abstract}

KEY WORDS - rhinotomy - chordomas • clivus - skull base tumor

Clival chordomas are malignancies of the central skull base. A combination of aggressive resection and highdose postoperative radiotherapy is frequently recommended. ${ }^{2,3}$ Many chordomas of the clivus extend posteriorly, distend or traverse the clival dura, and invaginate the brainstem (Fig. 1 left). The proximity of the tumor to the radiation-sensitive brainstem structures can limit the radiation dose that can be administered safely..$^{23}$ Removal of the tumor compressing the brainstem is thus a central goal of the surgery of clival chordomas.

Surgical approaches to clival chordomas include superior, lateral, and anterior routes. Subfrontal, transbasal routes approach clival tumors from above..$^{20}$ These approaches necessitate extensive cranial opening and significant retraction of the frontal lobe. Subtemporal routes approach clival tumors from the side ${ }^{21}$ and require retraction of the temporal lobe. Most subtemporal approaches

Abbreviations used in this paper: $\mathrm{CT}=$ computerized tomography; ICA = internal carotid artery; $\mathrm{MR}=$ magnetic resonance. offer only oblique access to the clival origin of the tumor and its anteroposterior expansion. Anterior, transfacial routes provide direct access to clival tumors along the most common axis of tumor growth. ${ }^{7,8,12,13}$

The clivus is coextensive with the nose in the superoinferior dimension (Fig. 1 left). Anterior transfacial approaches to the full clivus thus usually traverse the nasal cavity and its associated sinuses. ${ }^{9}$ Trans-nasal routes to the clivus are lengthened by the distance the nose extends from the midface. ${ }^{13}$ Pedicled rhinotomy provides a shorter surgical corridor to the clivus by disarticulating and laterally rotating nasal bone and cartilage away from the midface. ${ }^{11,22}$ The more shallow depth of field affords both proximity to and wider viewing angles of clival tumors (Fig. 2 left and right). ${ }^{10}$

The pedicled rhinotomy approach to the clivus is described. We present a series of 10 adult patients in whom this approach was used to remove clival chordomas invaginating the brainstem. The advantages and disadvantages of pedicled rhinotomy and the indications for its use are discussed. 


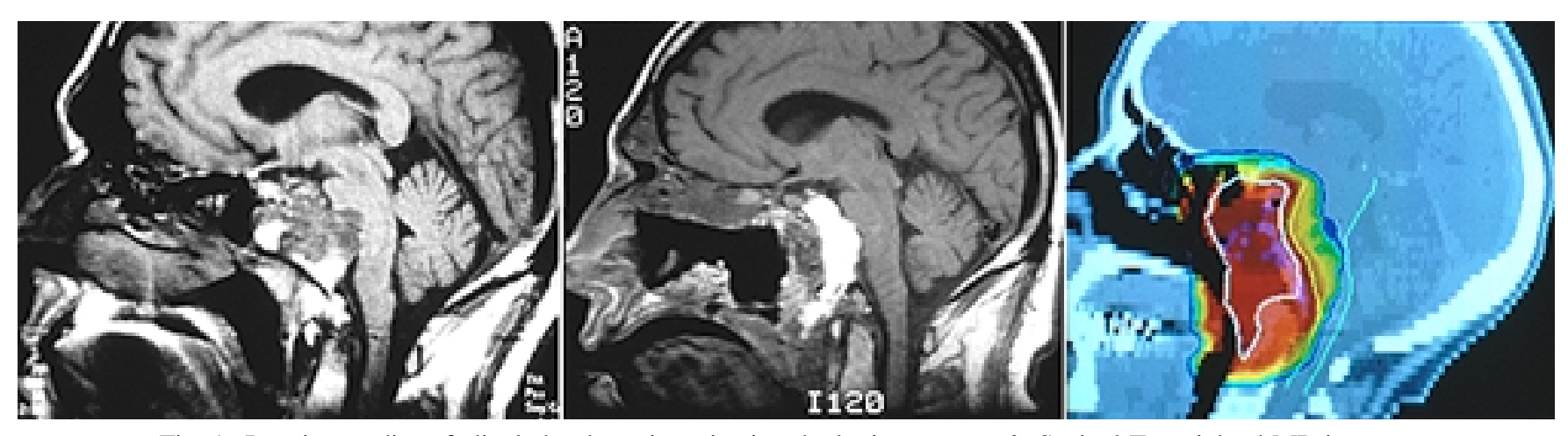

Fig. 1. Imaging studies of clival chordoma invaginating the brainstem. Left: Sagittal $\mathrm{T}_{1}$-weighted MR images revealing a large chordoma replacing the clivus and extending posteriorly and intradurally to invaginate the pons. Center: Image obtained following pedicled rhinotomy demonstrating removal of all but the superior tip of the tumor and relief of pontine invagination by tumor. The tumor has been replaced by fascia lata and skin grafts anteriorly and fat posteriorly. Right: Sagittally reconstructed CT scan, upon which proton radiation isodose lines have been superimposed, delineating the soft tissue of the closure positioned to both prevent cerebrospinal fluid leakage and separate the brainstem from the tumor bed to be irradiated.

\section{CLINICAL MATERIAL AND METHODS}

\section{Patient Population}

The pedicled rhinotomy approach was used to remove clival chordomas in 10 adult patients: six men and four women ranging in age from 30 to 61 years (mean 45.5 years). This was the first treatment in three $(30 \%)$ of the cases. Seven $(70 \%)$ of the 10 tumors had previously been surgically treated elsewhere; two patients had undergone two operations. Cumulatively, two orbitofrontal-temporal craniotomies and three transsphenoidal, one transpalatal, two transoral, and one transmandibular approach had been performed. Three tumors had recurred following both surgery and standard fractionated external beam irradiation (54 to $60 \mathrm{~Gy})$.

On presenting for this surgery, eight patients suffered headache; seven, diplopia; four, dysphagia; two, hearing loss; and two reported experiencing dysequilibrium. Five patients experienced complete palsy of one or more nerves to extraocular muscles, two were unilaterally deaf, and four had bulbar cranial nerve deficits. One patient was hemiparetic and one was severely ataxic.

All 10 patients underwent preoperative MR imaging that revealed a predominantly midline clival tumor extending posteriorly to abut or invaginate the brainstem. All tumors involved at least two of the three clival segments (the upper, middle, and lower). Four tumors were predominantly upper and middle, three were holoclival, and three were predominantly middle and inferior. Significant tumor-induced brainstem compression lateral to the petrous or cavernous ICA was a contraindication to the use of this approach.

\section{Preparation for Surgery}

In anticipation of using computer-assisted navigation, stereotactic fiducials are applied to the scalp and a highresolution gadolinium-enhanced MR image is obtained. Induction of general anesthesia follows orotracheal intubation. Antibiotic agents (vancomycin, ceftazidime, and metronizadole) are given. A lumbar subarachnoid drain is placed if opening of the dura is anticipated. The patient is then turned into a supine, semisitting position. MayfieldKeyes tongs are used to hold the head rotated to the right (facing the surgeon who is standing at the patient's right side) and slightly flexed or extended (depending on whether inferior or superior exposure, respectively, is more crucial). Intraoperative navigation equipment is positioned and the stereotactic fiducials are registered. Alternatively, an image-intesifying $\mathrm{C}$-arm is positioned as in a standard transsphenoidal operation.

Rarely, when palatal and pharyngeal function is compromised by tumor, a tracheostomy with gastrostomy is required. The hypopharynx and oropharynx are packed with moist gauze, and $0.05 \%$ oxymetazoline hydrochloride (Afrin) is applied to the nasal mucosa. The face is washed with $10 \%$ povidone iodine (Betadine) solution. The face, the left lower quadrant of the abdomen (for a subcutaneous fat graft), and the right thigh (for a splitthickness skin graft or fascia lata graft) are prepared in a sterile fashion and draped. Bilateral tarsorrhaphy is performed.

\section{Nasal Incision}

The nasal incision is marked after superficial Doppler ultrasonography is used to locate and verify the patency of the contralateral facial artery that will supply the pedicled nasal flap. The incision is infiltrated with $1 \%$ lidocaine (Xylocaine) with 1:100,000 epinephrine solution. The incision is carried through skin, subcutaneous tissue, and periosteum. It begins over the nasal bone along the side of the upper third of the nose and passes inferiorly in the skin crease marking the break point between the dorsum and lateral wall of the nose. It extends inferiorly over the upper and then the lower lateral cartilage, curving into the nasal sill along the base of the alae of the nose and columella at the base of the piriform aperture. The lip is not divided.

\section{Nasal Osteotomies}

Lateral nasal osteotomies are performed bilaterally at the junction between the nasal bone and the ascending process of the maxillary bone. The upper lateral cartilage 
is separated from the cartilaginous nasal septum, and the septum is disarticulated from the vomer and separated from the ethmoid bone. The ipsilateral nasal bone is not elevated with the lip but is separated from the base of the frontal bone. The lateral cartilages are separated from the nasal spine by a transcolumellar cut through the medial crus of each lateral cartilage. The anterior cartilaginous septum is divided from the maxillary crest. It thus remains part of the nasal pedicle.

\section{Nasal Dissection}

A stab incision is placed just anterior to the inferior turbinates. This permits a subperiosteal dissection that preserves both the facial artery and the attachment of the nasal bone to the maxilla despite the osteotomies. The ipsilateral lateral nasal bone and upper lateral cartilage are retracted laterally. The nasal flap, with the attached nasal septum and contralateral lateral nasal bone, is rotated to the side opposite the incision (Fig. 2 left). Compromise of the vascular supply to the flap is avoided by placing a rolled sponge beneath the fold. The flap is returned to its anatomical position for 5 minutes every hour to ensure adequate perfusion.

\section{Nasal and Sinus Openings}

Remnants of the nasal septum are removed, except for a narrow strip along the roof of the nasal cavity containing the olfactory epithelium. Removal of the turbinates and the medial walls of the maxillae improves the view of the structures lying posterolaterally, the ICA and medial cavernous sinus above, and the jugular foramen and lower cranial nerves below. The middle turbinates are removed, and the ethmoid air cells are opened widely. The medial wall of the maxillary sinus, including the inferior turbinates, is removed. The lacrimal duct is divided. An ipsilateral maxillectomy is performed by removing the anteri-

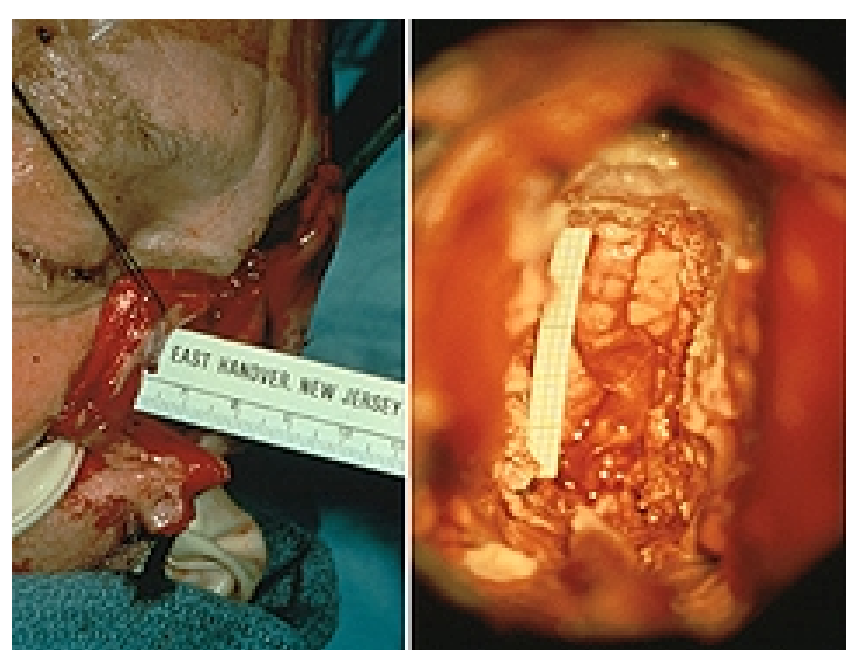

Fig. 2. Exposure in a pedicled rhinotomy. Left: Intraoperative photograph. Disarticulation and rotation of the nose provide a short, wide, and direct route to the clivus. Right: Photograph of a cadaveric specimen showing that the clival exposure and potential opening extends 5 to $6 \mathrm{~cm}$ superoinferiorly and 2 to $4 \mathrm{~cm}$ mediolaterally. or wall as far laterally as the foramen of the infraorbital nerve. The contralateral maxillary sinus is opened medially. The soft palate is retracted superiorly by using a Penrose drain that is passed through the mouth (Fig. 2 left).

\section{Clival Exposure}

Superiorly, the rostrum and anterior face of the sphenoid sinus are removed. The sinus is exenterated of its mucosa. The tuberculum sellae and the anterior wall and floor of the sella are identified. When normal anatomy of the clivus is distorted by tumor, computer-assisted navigation or the image intensifying C-arm fluoroscopy can be useful in locating important structures. The intraoperative position and orientation of the septations of the sphenoid sinus are useful independent guides in determining one's position in the mediolateral dimension. Inferiorly, the posterior pharyngeal mucosa is incised in the midline and dissected from underlying clivus or tumor. The preclival periosteum becomes quite densely attached along a longitudinal groove that runs superior to inferior along each side of the midline clival convexity.

\section{Clival Opening}

The normal clivus is 10 to $15 \mathrm{~mm}$ thick. A high-speed air drill is used to reduce it in size to a thin cortical remnant that can be fractured anteriorly with a small-curved curette or removed using a thin-footed Kerrison rongeur. The choice of location and size of the opening is guided by the neuronavigational depiction of the extent of the tumor within and beneath the clivus. Orientation in the anteroposterior dimension is best determined by exposing normal dura superior to the tumor.

The clival bone can then be removed as widely as is needed bilaterally as one proceeds from superior to inferior along the anterior surface of the dura or tumor. Bone bleeding is controlled by the application of wax, and venous channels are either coagulated or occluded with Surgicel. The venous channels of the clival dura interconnect with marginal and petrosal sinuses, and contact should be avoided when possible.

Removal of the clivus is continued laterally until the tumor is adequately exposed within the constraints imposed by the ICAs, the jugular foramina, the hypoglossal canals, and the need to retract densely adherent soft tissue at the depths of the field. Often much of the tumor is removed as the central clivus is being resected.

The limits of the exposure include the tuberculum sellae superiorly and the junction of the odontoid with the body of C-2 inferiorly (Figs. 1 center and 2 right). Superiorly, lateral exposure is limited to a total of $20 \mathrm{~mm}$ by the cavernous ICA on each side (Fig. 3 upper left and right). In the midportion of the clivus, bone can be removed out to the junction of the petrous and cavernous segments of the ICA, separated by $20 \mathrm{~mm}$ superiorly and at least $30 \mathrm{~mm}$ inferiorly (Fig. 4 left). In the lower clivus, lateral removal of bone is restricted to a total width of approximately $40 \mathrm{~mm}$ by the dense attachments of retropharyngeal soft tissue, and, near the foramen magnum, the hypoglossal nerves again restrict the opening to $30 \mathrm{~mm}$ in width (Fig. 3 lower left and right). This opening into the clivus is adequate to access the anterior medulla and pons, the vertebral arteries bilaterally, the vertebrobasilar junc- 

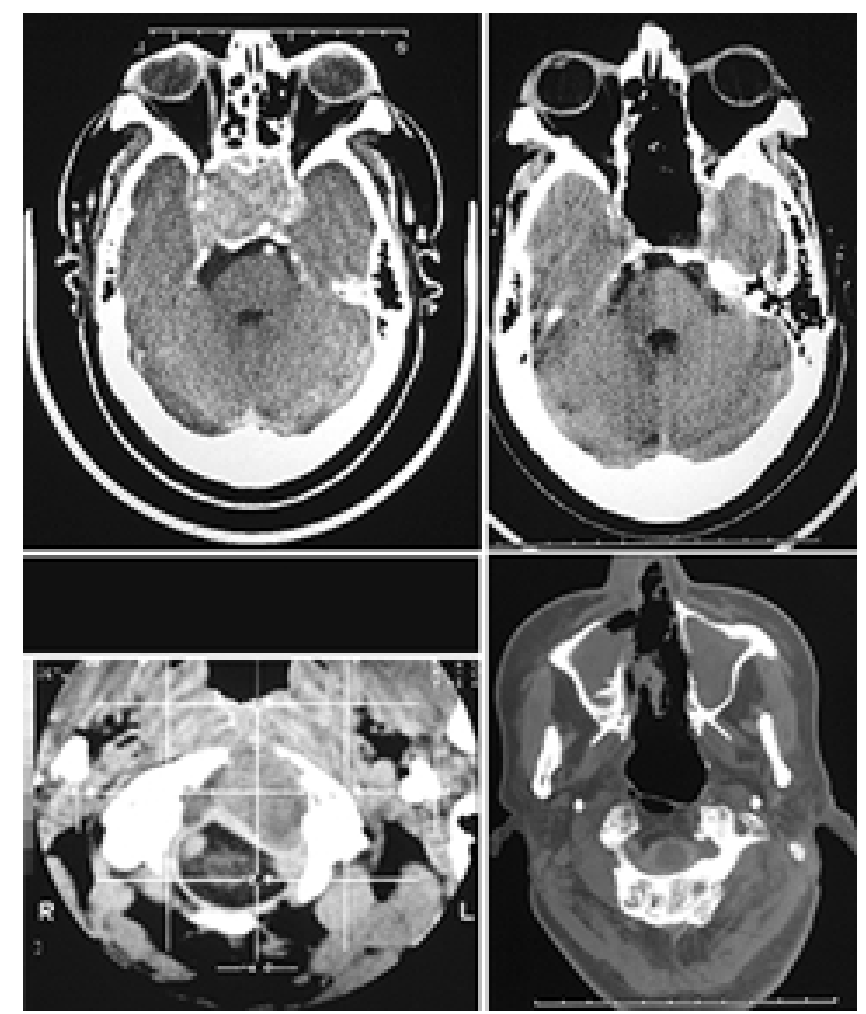

Fig. 3. The surgical corridor. Preoperative (upper left) and postoperative (upper right) axial CT scans demonstrating that the surgical corridor of the pedicled rhinotomy can be expanded by tumor-induced lateral displacement of the cavernous sinuses and ICAs. Erosion of bone by tumor (lower left) also facilitates obtaining the wide exposure required for complete tumor resection (lower right).

tion, and the inferior half of the basilar artery (Figs. 2 right and 4 left).

\section{Tumor Removal}

Centrally, after the clivus and any portion of tumor infiltrating it have been removed, the posterior aspect of the tumor remains attached to attenuated dura. Although it is tempting to excise the tumor nodule and an annulus of surrounding dura, it is best to continue piecemeal removal of the tumor in an effort to preserve as much tumor-free dura as possible. Often the dura is intact but markedly attenuated; progressive centripetal dissection of tumor from dura will usually define a surprisingly small dural defect. Dura that is grossly infiltrated by tumor must be removed, but care must be taken to preserve the intact arachnoid beneath it. If dura must be more widely opened to resect the intradural tumor, it should be incised longitudinally. Opening into basal venous sinuses should be avoided if possible. Bleeding can be controlled by coagulation in which a right-angled bipolar forceps is used or the sinuses are packed with Surgicel. The intradural tumor is often soft and can be aspirated through a fine fenestrated sucker. The risk of injury to the vertebrobasilar arteries and their perforating branches, both sixth cranial nerves,

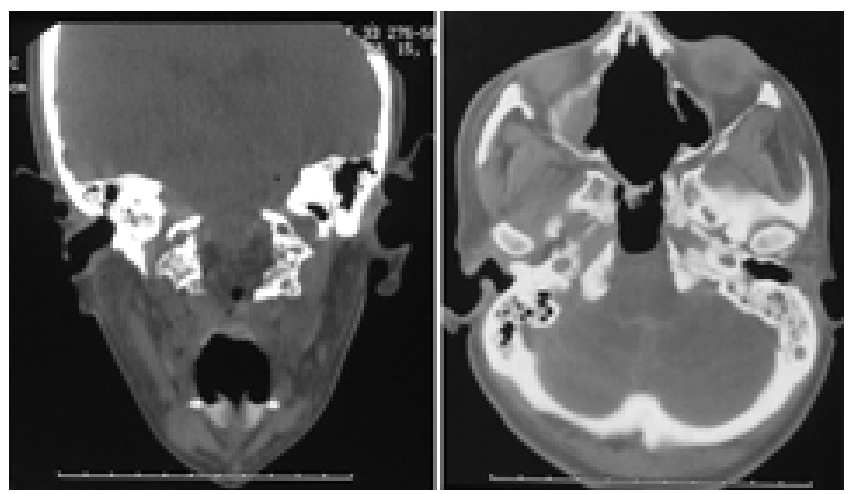

Fig. 4. Limits of exposure obtained via a pedicled rhinotomy. Postoperative coronal (left) and axial (right) CT scans demonstrating that the width of surgical corridor achieved is limited to the distance between the intrapetrous and intracavernous ICAs.

and the anterior surface of the brainstem is reduced by preserving the arachnoid plane whenever possible.

\section{Closure Procedure}

Closure begins with repair of the dural opening. Primary microsurgical suturing is preferred, but this is often precluded by the extent to which the tumor has destroyed the dura. In some cases, it is possible to sew a patch of fascia lata to the edges of the dural opening superiorly and inferiorly. The irregularity of the opening and the narrowness and depth of the exposure often hamper this effort. The exposed dura and the dural graft are then covered with layers of abdominal fat, fibrin glue, and fascia lata (Fig. 1 center and right). Either longitudinally pedicled flaps of nasopharyngeal mucosa or a split-thickness (0.12inch) skin graft is placed over the opening in the clivus. This layer is held in place by packing the posterior pharynx with bacitracin-impregnated iodoform gauze.

When medial maxillectomy has been performed, the lacrimal duct is repaired with Silastic tubing. Gauze packed within a finger cot is placed along the soft palate. The pedicled nasal flap is rotated back into position. The cartilaginous nasal septum is sutured to the remnant of septal cartilage along the maxillary crest. The external incisions are closed in two layers with No. 5-0 clear polydioxanone suture subcutaneously and No. 6-0 nylon suture in skin. The transcolumellar incision is closed with No. 6-0 nylon suture. The medial crus is reapproximated, and a columellar strut is positioned behind it. Clear polydioxanone No. 5-0 sutures and Surgicel impregnated with bacitracin are used to stabilize the anterior cartilaginous complex and nasal bone. An external cast is applied, and the tarsorrhaphy sutures are removed.

Exposure and closure take 1 to 2 hours for each procedure. The time for tumor resection and reconstruction of the dura ranges from 2 to 4 hours. The average estimated blood loss for the opening and closing is approximately $300 \mathrm{ml}$.

Postoperatively, the lumbar drain, the nasal splint, and the nylon sutures are removed on the 5th day and nasal packing on the 14th day. Intravenous antibiotics are continued for 2 weeks. The lacrimal tube is removed after 2 
Pedicled rhinotomy for clival chordomas

months. The patient is instructed to irrigate the nasal cavity daily to prevent mucus accumulation and crusting.

\section{RESULTS}

Operative findings included dural involvement by tumor in all cases. In eight cases, the tumor had traversed dura to the extent that its removal would expose arachnoid; in six cases, the arachnoid was breached. All targeted dural and intradural tumor was completely removed in eight of the 10 cases. The dura was repaired by fascia lata reinforced with skin graft in seven cases and with mucosal flaps in three cases. Estimated blood loss ranged from 500 to $1000 \mathrm{ml}$. The average duration of surgery was approximately 6.5 hours, including approximately 1.5 hours for opening, 3.5 hours for tumor resection, and 1.5 hours for closure.

Postoperative MR imaging confirmed that tumor abutting or compressing the brainstem was completely removed via the pedicled rhinotomy in eight cases. In one patient a subsequent subtemporal craniotomy was required for resection of suprasellar tumor, compressing the optic chiasm, which could not be removed through the rhinotomy. In one patient who had previously undergone radiotherapy, resection of tumor adjacent to the brainstem was incomplete. Removal of tumor allowed us to perform proton beam irradiation in the seven cases in which radiotherapy had not previously been conducted.

No patient sustained any new neurological deficit. Seven of eight patients experienced improvement of their headache. There was improvement of diplopia, dysphagia, and hemiparesis in one case each. One patient developed a postoperative cerebrospinal fluid leak and bacterial meningitis; these were successfully treated with placement of a shunt and antibiotics. One patient sustained infection of a lacrimal sac, which resolved with drainage and antibiotics. In one patient a small columellar tear required suture repair. No patient expressed dissatisfaction with the cosmetic result.

\section{DISCUSSION}

Clival chordomas are challenging lesions to resect. Access is restricted by the hemispheres superiorly, the nasooropharnyx anteriorly, the cavernous sinuses, temporal lobes, and petrous bones laterally, and the brainstem posteriorly. Clival chordomas have been approached from each of the following routes: subfrontal and transbasal; transnasal, transpalatal, transmaxillary, transoral, and transmandibular; transsylvian, subtemporal, and transpetrous; and suboccipital. . $^{1,46-8,13,15,21}$ The decision to use one of these approaches should be based on four criteria: full exposure of the targeted tumor, safety from injury to neural and vascular structures, security of dural repair, and cosmesis.

An anterior approach is often preferred for lesions located anterior to the hemispheres and brainstem. The major advantage is that brain retraction is avoided. In most anterior approaches the cavities of the nose, paranasal sinuses, mouth, and pharynx are used to view poste-

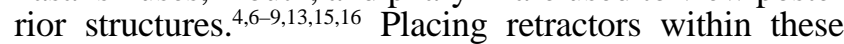
cavities opens a wide surgical corridor with a more shallow depth of field than would otherwise be obtained (Fig.

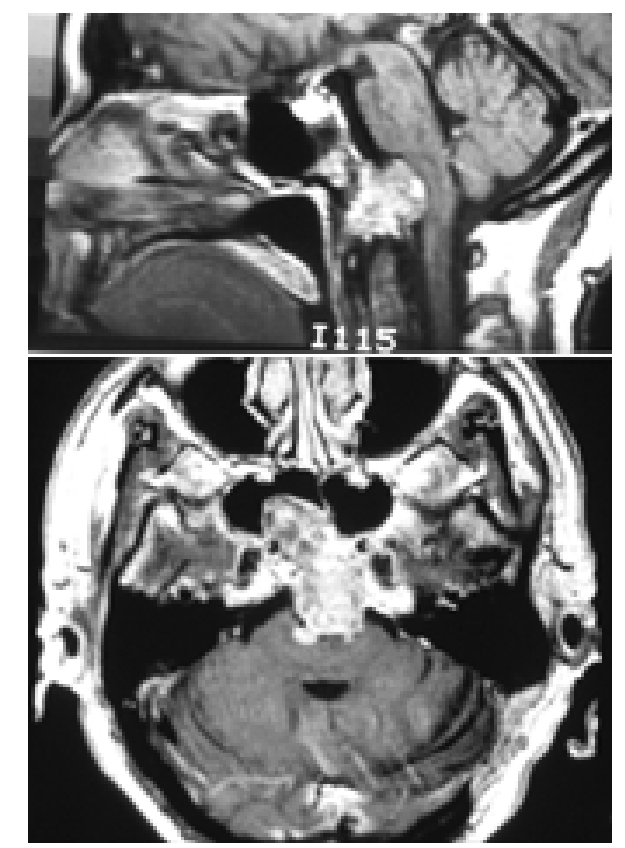

Fig. 5. Clival chordoma for pedicled rhinotomy. Preoperative sagittal (upper) and axial (lower) contrast-enhanced MR images demonstrating a chordoma appropriate for pedicled rhinotomy. The tumor involves the middle and lower clivus (upper) at levels difficult to access completely via either transnasal or transoral routes alone. The tumor invaginating the brainstem lies within the intercarotid surgical corridor. The extent of midline invagination (lower) poses greater challenge to a lateral than to a direct anterior approach.

2 left). In working through air-filled cavities the surgeon avoids the more extensive dissection required when traversing solid bone or soft tissue.

In traversing the face and the nasal and oropharyngeal cavities, however, there are distinct disadvantages as well. The contaminated field risks soft-tissue infections, osteomyelitis, brain abscess, and meningitis. Although prophylactic antibiotics help reduce these risks, they are no substitute for preservation of vascular supply to soft tissue or effective dural closure. The one case of meningitis in this series attests to the seriousness of this risk. When pedicled rhinotomy is used to treat an aneurysm, this risk is heightened due to compromise of closure by the aneurysm clip or by communicating hydrocephalus subsequent to subarachnoid hemorrhage. ${ }^{17}$ Chronic sinusitis and intranasal crusting can also be problematic. The risks of facial incisions and osteotomies of the facial skeleton are scarring and disfigurement that may be cosmetically and psychologically injurious to the patient. The risk of ischemic injury is associated with the use of a pedicled flap, but this can be minimized by intraoperative attention to flap perfusion. Visceral functions of olfaction, speech, swallowing, and airway management are also vulnerable.

The direct anterior-posterior approach performed in the pedicled rhinotomy is ideal for many clival chordomas because the majority of the tumors grow preferentially in the anteroposterior dimension (Fig. 5). The surgical corridor is aligned with this direction of preferential growth and often encompasses all the chordoma that must be 
removed to optimize the geometrical configuration of the postoperative radiation field (Fig. 1 right). Lateral intradural approaches offer poor access to tumors within the clivus, and even extradural lateral approaches are unable to remove tumors that have extended anteriorly. Although the lateral dimension of the pedicled rhinotomy exposure is limited at clival depth to the distances between the petrous and cavernous segments of the ICAs, the relatively shallow depth of field permits lateral angulation of the microscope to achieve exposure of a wider field beyond this restriction. Furthermore, when lateral extension occurs, tumor expansion often laterally displaces the structures limiting lateral exposure (Fig. 3).

Additional attractive features of the pedicled rhinotomy approach relative to the alternative, more extensive anterior approaches include avoidance of an incision into the philtrum and lip, avoidance of division of either hard or soft palate, and freedom from the challenge of having to reapproximate the facial skeleton. Also avoided are the extensive facial osteotomies of the extended maxillotomy approach. ${ }^{1,6}$ In contrast to an extended maxillectomy, there is no need for intramaxillary fixation; metal plates that might degrade the quality of postoperative images are not required. In comparison with the transpalatal approach, there is no risk of palatal dysfunction and blood loss is relatively low. The lateral nasal incision of the pedicled rhinotomy, although it usually heals into a very thin linear scar, is still less desirable than the cosmetically covert sublabial and intranasal incisions of facial degloving. Facial degloving, however, when not followed by the nasal osteotomies of the pedicled rhinotomy, fails to achieve the same shallowness of field. ${ }^{5,14,19}$

When required by tumors that extend beyond the limits of a pedicled rhinotomy, this procedure may be combined with other approaches. The nasal cavity and associated paranasal air sinuses are limited superiorly by the floor of the anterior cranial fossa. Lateral rhinotomy can be combined with craniotomy for tumors that extend superiorly. ${ }^{4,18}$ It can also be combined with sublabial incisions and maxillectomy for tumors with greater lateral extension. ${ }^{1,6}$

In conclusion, the pedicled rhinotomy offers shallowfield exposure of the midline anterior skull base without requiring division of hard or soft palates, which is similar to the combination of transoral and transnasal, transsphenoid routes. ${ }^{13,15,16}$ The upper third of the clivus is best approached via a transnasal, transsphenoidal approach ${ }^{13}$ or a transethmoid, transsphenoidal route ${ }^{8}$ that avoids the extensive dissections of a pedicled rhinotomy. The lower third of the clivus down to the $\mathrm{C} 1-2$ junction is best approached via a transoral route. ${ }^{15,16}$ For exposure of tumor lateral to the ICA or in the medial petrous bone, a lateral, as well as an anterior, approach is often needed. ${ }^{20}$

\section{References}

1. Anand VK, Harkey HL, Al-Mefty O: Open-door maxillotomy approach for lesions of the clivus. Skull Base Surg 1:217-225, 1991

2. Austin-Seymour M, Munzenrider J, Goitein M, et al: Fractionated proton radiation therapy of chordoma and low grade chondrosarcoma of the base of the skull. J Neurosurg 70: 13-17, 1989

3. Benk V, Liebsch NJ, Munzenrider JE, et al: Base of skull and cervical spine chordomas in children treated by high-dose irradiation. Int J Radiat Oncol Biol Phys 31:577-581, 1995

4. Blacklock JB, Weber RS, Lee YY, et al: Transcranial resection of tumors of the paranasal sinuses and nasal cavity. J Neurosurg 71:10-15, 1989

5. Casson PR, Bonanno PC, Converse JM: The midface degloving procedure. Plast Reconstr Surg 53:102-103, 1974

6. Cocke EW, Robertson JH, Robertson JT, et al: The extended maxillotomy and subtotal maxillectomy for excision of skull base tumors. Arch Otolaryngol Head Neck Surg 116:92-104, 1990

7. Crockard AH: Transoral approach to intra/extradural tumors, in Sekhar LN, Janecka IP (eds): Surgery of Cranial Base Tumors. New York: Raven Press, 1993, pp 225-234

8. Crumley RL, Gutin PH: Surgical access for clivus chordoma. The University of California, San Francisco, experience. Arch Otolaryngol Head Neck Surg 115:295-300, 1989

9. deFries HO, Deeb ZE, Hudkins CP: A transfacial approach to the nasal-paranasal cavities and anterior skull base. Arch Otolaryngol Head Neck Surg 114:766-769, 1988

10. Harsh GR, Joseph MP, Swearingen B, et al: Anterior midline approaches to the central skull base. Clin Neurosurg 43: 15-43, 1996

11. Joseph M: Pedicled rhinotomy for exposure of the clivus, in Schmidek HH, Sweet WH (eds): Operative Neurosurgical Techniques, ed 3. Philadelphia: WB Saunders, 1995, pp 469-475

12. Krespi YP, Sisson GA: Transmandibular exposure of the skull base. Am J Surg 148:534-538, 1984

13. Laws ER Jr: Transsphenoidal surgery for tumors of the clivus. Otolaryngol Head Neck Surg 92:100-101,1984

14. Maniglia AJ: Indications and techniques of midfacial degloving. A 15-year experience. Arch Otolaryngol Head Neck Surg 112:750-752, 1986

15. Menezes AH, VanGilder JC: Transoral-transpharyngeal approach to the anterior craniocervical junction. Ten-year experience with 72 patients. J Neurosurg 69:895-903, 1988

16. Miller E, Crockard HA: Transoral transclival removal of anteriorly placed meningiomas of the foramen magnum. Neurosurgery 20:966-968, 1987

17. Ogilvy CS, Barker FG, Joseph MP, et al: Transfacial transclival approach for midline posterior circulation aneurysms. Neurosurgery 39:736-741, 1996

18. Panje WR, Dohrmann GJ, Pitcock JK, et al: The transfacial approach for combined anterior craniofacial tumor ablation. Arch Otolaryngol Head Neck Surg 115:301-307, 1989

19. Price JC: The midfacial degloving approach to the central skullbase. Ear Nose Throat J 65:174-180, 1986

20. Sekhar LN, Sen C, Snyderman CH, et al: Anterior, anterolateral, and lateral approaches to extradural petroclival tumors, in Sekhar LN, Janecka IP (eds): Surgery of Cranial Base Tumors. New York: Raven Press, 1993, pp 157-224

21. Sen CN, Sekhar LN, Schramm VL, et al: Chordoma and chondrosarcoma of the cranial base: an 8-year experience. Neurosurgery 25:931-941, 1989

22. Swearingen B, Joseph M, Cheney M, et al: A modified transfacial approach to the clivus. Neurosurgery 36:101-105, 1995

23. Urie MM, Fullerton B, Tatsuzaki $\mathrm{H}$, et al: A dose response analysis of injury to cranial nerves and/or nuclei following proton beam radiation therapy. Int J Radiat Oncol Biol Phys 23: 27-39, 1992

Manuscript received January 30, 2001.

Accepted in final form February 15, 2001.

Address reprint requests to: Griff Harsh, M.D., Stanford Brain Tumor Center, Stanford Medical Center, 300 Pasteur Drive, R227, Stanford, California 94304. 